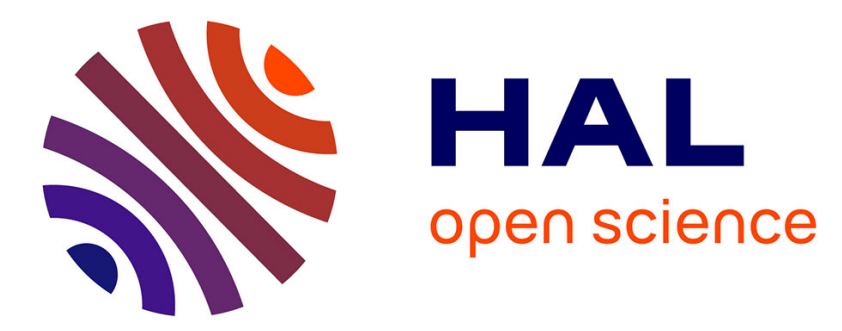

\title{
Note sur les tablettes de cire de Dijon Élisabeth Lalou
}

\section{To cite this version:}

Élisabeth Lalou. Note sur les tablettes de cire de Dijon. Matériaux du livre médiéval, pp.231-236, 2010, 10.1484/m.bib-eb.3.4504 . hal-01801525

\section{HAL Id: hal-01801525 \\ https://hal.science/hal-01801525}

Submitted on 28 May 2018

HAL is a multi-disciplinary open access archive for the deposit and dissemination of scientific research documents, whether they are published or not. The documents may come from teaching and research institutions in France or abroad, or from public or private research centers.
L'archive ouverte pluridisciplinaire HAL, est destinée au dépôt et à la diffusion de documents scientifiques de niveau recherche, publiés ou non, émanant des établissements d'enseignement et de recherche français ou étrangers, des laboratoires publics ou privés. 


\title{
Note sur les tablettes de cire de Dijon
}

\author{
Élisabeth LALOU
}

Cette note présente un extrait d'une enquête encore en cours: d'une part parce que les recherches sur ce livre de tablettes de cire menées dans le cadre du GDR « Matériaux du livre médiéval »n'ont pas encore livré leurs résultats définitifs; d'autre part, parce que l'édition du manuscrit dont je vais vous parler n'est pas achevée ${ }^{1}$. Mais il importait avant tout que le travail sur les tablettes de cire, prévu dans le programme du GDR soit exposé au cours de ce colloque « bilan », c'est pourquoi je présente aujourd'hui ce volume de tablettes et les questions qu'il convient de se poser à son sujet: contenu, support, particularités.

\section{DESCRIPTION}

Aux Archives départementales de la Côte-d'Or à Dijon, sous la cote 11 H 1165*, est conservé dans le fonds des archives de l'abbaye de Cîteaux un livre de comptabilité ${ }^{2}$ constitué de feuillets de bois et de cire daté de 1321-1325 (figures 1, 2 et 3 ).

Il est constitué de douze feuillets, onze portent de la cire au recto et au verso, le douzième porte de la cire au recto et son verso est la couverture du volume. Il manque le feuillet de couverture du début et probablement un ou deux autres feuillets. On doit les rapprocher de deux autres feuillets pro-

Figure 1 - Arch. dép. Côté d'Or, 11 H 1165 Couverture du volume de tablettes (C) $\mathrm{CG} 21 / \mathrm{F}$. Petot $/ 2010$

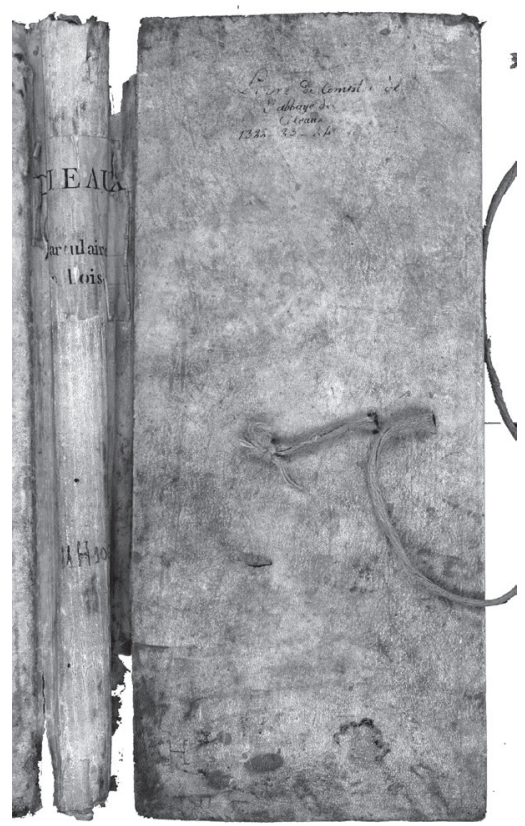

1. Elle devrait prendre place dans la collection Monumenta palaeographica Medii Aevi.

2. Jean Rigault, Guide des archives de la Côte d'Or, Dijon, 1984, p. 236-237; Ferdinand Claudon, Notice sur les archives de la Côte d'Or, Dijon, 1911 p. 91 ; J. FARENCE et P. ArABEYRe, L'écriture au Moyen Âge en Bourgogne IXe-XVe siècles, Dijon, 1988. 
venant aussi d'un volume de tablettes de cire de Cîteaux qui sont conservés à Londres ${ }^{3}$ et à Lyon $^{4}$.

Le registre de cire de Dijon et ces deux feuillets sont des tablettes « classiques » du XIV siècle. Les feuillets mesurent $155 \times 360 \mathrm{~mm}^{5}$.

\section{L'ÉCRITURE}

Elle est parallèle au grand côté des tablettes. Le registre se tenait donc comme un ordinateur portable. La page comporte trois colonnes. L'écriture est d'un module assez gros. L'écrit est cancellé puisqu'il s'agit de brouillons qui étaient ensuite recopiés sur du parchemin. Ces brouillons sont les comptes du boursier de Cîteaux.

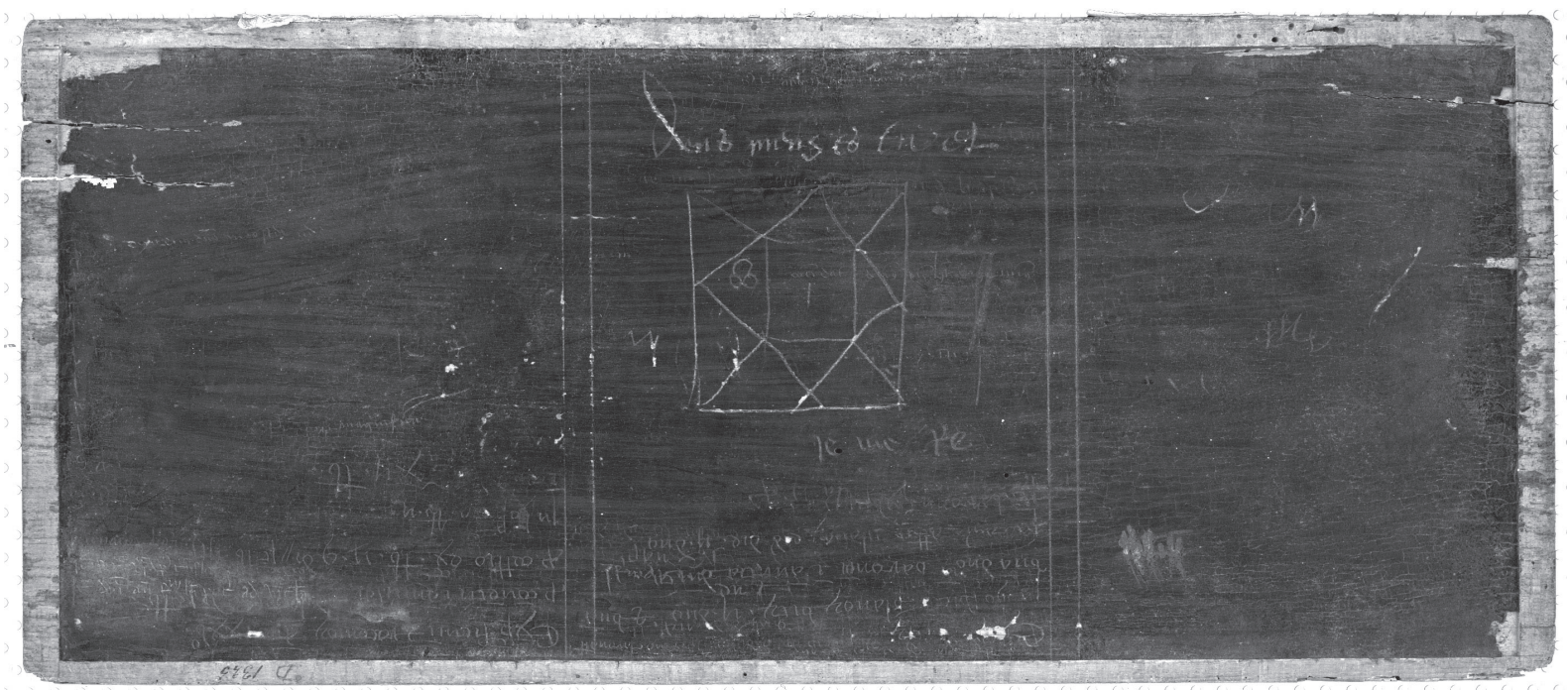

Figure 2 - Arch. dép. Côte d’Or, 11 H 1154, f. 5D verso

Schéma dessiné à l'envers de la page dont on distingue les lignes d'écriture en bas de la photo (C) CG21/F. Petot/2010

Nous avons d'ailleurs conservé un registre papier de comptes de l'ordre cistercien (11 H 1160*) mais plus tardif(1337-1402). Ce manuscrit s'ouvre sur des comptes généraux de l'ordre (1337-1347) et se poursuit par les comptes du boursier qui commencent en 1342 (f. 32245). Les autres registres de comptes du boursier sont encore plus tardifs : 1489-1501 pour le

3. London, British Library, add. 33215. Henri Omont, « Sur des tablettes de cire du Musée britannique », dans Comptes rendus des séances de la Société des Antiquaires de France, t. 50bis, 1889, p. 283, planches (une tablette, 2 faces, 1323-1324)

4. Lyon, Bibl. mun. 1736 (1702), Collection lyonnaise de Fac similés, pl. II (une tablette, 2 faces ; $351 \times 155$ ).

5. Le feuillet conservé à Londres est cassé et fragmentaire : les mesures sont donc aléatoires $(122 \times 242)$ mais la présentation et la mise en page sont identiques au registre de Dijon. 


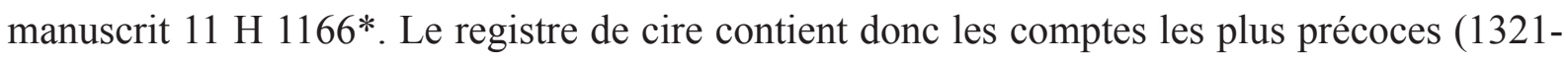
1325) du boursier de Cîteaux.

Le contenu de ces comptes n'a qu'un intérêt annexe ici. Ils contiennent des recettes et des dépenses stipulées en argent. Notre homme touche les revenus de certains domaines: étangs, bois, et surtout vignobles. Il fournit des sommes d'argent pour diverses dépenses : rétribution des travaux effectués dans différentes granges, dépenses de logement et de bouche lors de chapitres tenus à Dijon, déplacements de l'abbé ou de lui-même qui se rend « aux foires » etc. Ces comptes concernent Cîteaux mais aussi Morimond ${ }^{6}$ et Clairvaux.

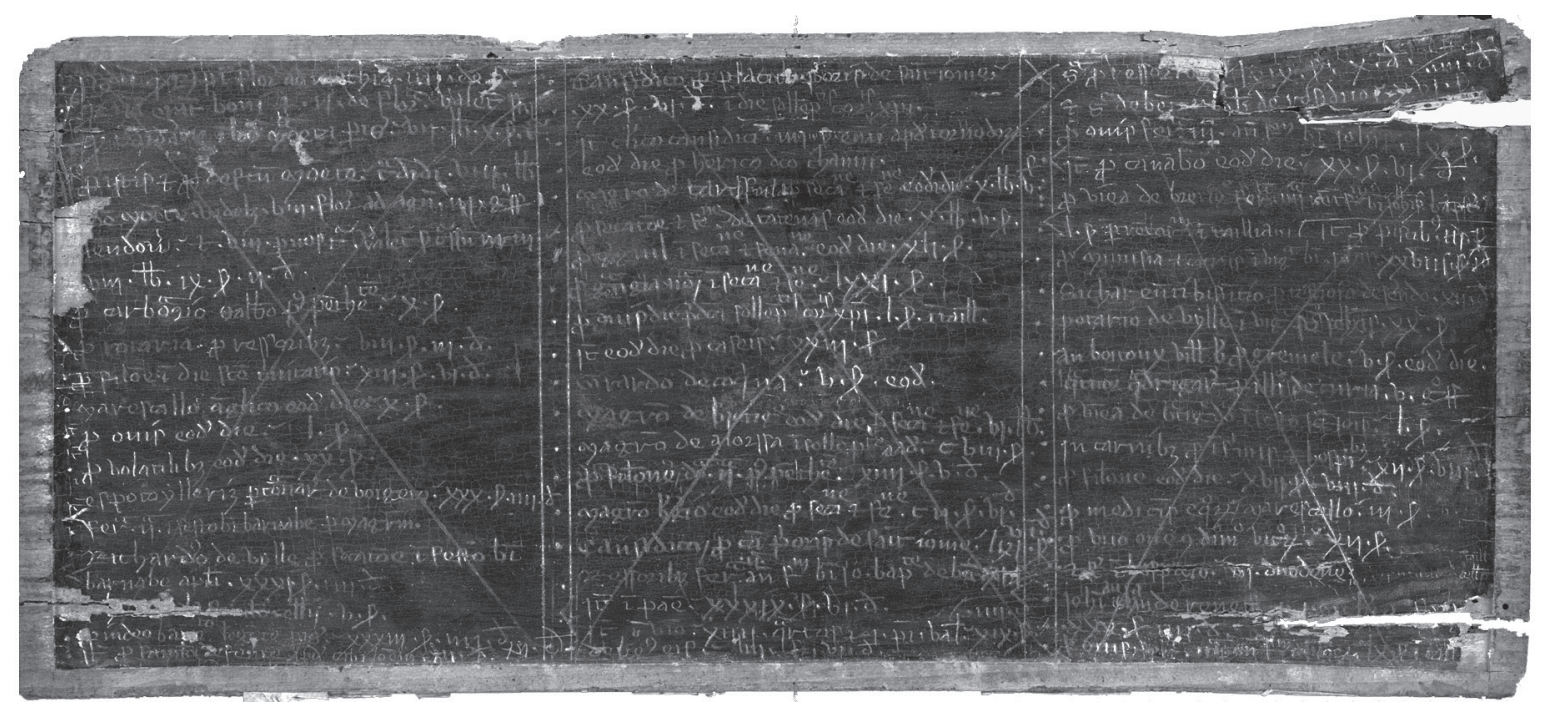

Figure 3 - Arch. dép. Côte d'Or, 11 H 1154, f. 8HJ verso

Mise en page identique dans tout le volume : trois colonnes. L'écriture a été cancellée après copie sur une feuille de parchemin. Noter la fragilité de cette page

\section{LA RELIURE}

Puisqu'il s'agit d'un avant-propos à la séance sur la reliure prévue dans ce colloque, je voudrais insister sur la façon dont étaient reliés les livres de tablettes, celui qui nous occupe aujourd'hui, comme les autres volumes que j'ai pu examiner. Les feuillets étaient réunis entre eux par des languettes de parchemin de 3 à $4 \mathrm{~cm}$ de large: ces languettes étaient insérées dans des fentes ménagées dans la tranche de la planchette. Elles maintenaient les feuillets bien serrés les uns contre les autres. Souvent un morceau de parchemin recouvrait la tranche du volume 
et donnait l'aspect d'un registre banal en matériau souple au volume de tablettes ${ }^{7}$. Ce mode de reliure est celui que 1'on trouve parfois dans $1^{\prime}$ Antiquité ${ }^{8}$ pour rapprocher des feuillets de bois mais il est loin de constituer alors la majorité : en effet les tablettes antiques sont maintenues ensemble le plus souvent par un morceau de ficelle ou encore des sortes de gonds. Je ne crois pas me tromper en identifiant cette reliure des tablettes de Dijon à un modèle de reliure très répandu à l'époque médiévale. Signalons aussi que dans ce volume la tranche ne comporte pas de marques utilisées parfois pour faciliter l'alignement et l'ordre des feuillets.

\section{MATÉRIAUX}

\section{La cire}

Les planchettes sont creusées pour recevoir une couche de cire noire d'environ un millimètre d'épaisseur. La couche de cire est peu épaisse: le registre a déjà été utilisé une ou deux fois avant la date du compte aujourd'hui conservé. On effaçait en effet la cire en l'aplatissant avec l'autre extrémité du stylet qui servait à tracer les lettres. Parfois on " recirait » les tablettes: c'est ce que l'on trouve dans les comptes de la ville de Senlis ${ }^{9}$.

Dans une étude allemande réalisée en 1968 par Reinhard Bull, Ernst Moser et Hermann Kuhn ${ }^{10}$, est exposé le résultat des analyses du contenu de la cire d'une trentaine de volumes de tablettes de cire conservés en Allemagne. Il n'a pas été encore fait d'analyse du contenu de la cire du volume de Dijon mais on peut sans doute penser que les résultats de l'étude allemande sont proches de ce que l'on trouvera lors de l'analyse des volumes de tablettes français.

Ce matériau est constitué d'un mélange de cire d'abeille, de résine et de poix. On y ajoutait aussi du noir de fumée pour obtenir la couleur noire. On pouvait rendre le mélange plus épais, en mélangeant différents produits à la cire. La consistance permettait d'écrire plus aisément et d'éviter que le trait ne s'effondre à cause du peu de tenue du produit.

Nous avons conservé des recettes antiques ${ }^{11}$ permettant d'obtenir ce mélange qui est dans la plupart des cas de couleur noire. D'autres couleurs étaient utilisées : le vert (dans des carnets de petit format qui nous sont parvenus), le rouge (dans des carnets d'ivoire) et parfois le jaune

7. C'est le cas du registre de Dijon. Toutefois il n'est pas certain que le parchemin soit d'origine.

8. Cf. Élisabeth LaLou (éd.), Les tablettes à écrire de l'Antiquité à l'époque moderne. Actes du colloque de Paris, octobre 1990, Turnhout, 1992 (Bibliologia, 12).

9. É. Lalou, Les tablettes à écrire de l'Antiquité...; ID., « Les tablettes de cire médiévales », Bibliothèque de l'École des chartes 147, 1989, p.123-140; ID., Les comptes sur tablettes de cire de la chambre aux deniers de Philippe III le Hardi et Philippe IV le Bel (1282-1309), R.-H. BAUTIER (dir.), Paris, 1994 ; ID., Les comptes sur tablettes de cire de Jean Sarrazin, chambellan de saint Louis, Turnhout, 2003 (Monumenta palaeographica Medii Aevi), 205 p.

10. Reinard Bull, Ernst Moser, Hermann KuHn, «Wachs als Beschreib- und Siegelstoff, Wachsschreibtafeln und ihre Verwendung », dans Vom Wachs-Hoechster, Beiträge zur Kenntnis der Wachse, Frankfurt, 1968, p. 785-894.

11. R. Bull, E. Moser, H. Kunn, «Wachs als Beschreib- und Siegelstoff... », p. 818 : « Recipe tres partes cere, quartam partem picis, et quintam partem cynober et ceram cum pice pone in cacabum...» (recette pour le sceau). 
ou plutôt la couleur de la cire vierge, comme dans un carnet du XVII siècle de la BnF ${ }^{12}$. Il serait intéressant d'analyser ${ }^{13}$ ces cires de différentes couleurs pour savoir si elles étaient identiques à celles utilisées dans les sceaux. L'analyse de la cire n'était pas prévue dans le projet initial du GDR mais l'idée s'en est imposée peu à peu et reste un des objectifs intéressant de recherche.

\section{Le bois}

Nous avions prévu en revanche d'analyser et identifier le bois d'un certain nombre de tablettes de cire ${ }^{14}$. Les hypothèses les plus diverses ont été émises sur le bois des tablettes de Jean Sarrazin, chambellan de Saint-Louis, conservées aux Archives nationales. Natalis de Wailly pensait qu'elles étaient en platane ; j'ai moi-même cru reconnaître du hêtre ${ }^{15}$. Une analyse scientifique a finalement identifié de l'érable.

J'en étais restée là, face à ce résultat inattendu jusqu'à une découverte récente: au début du XIVe siècle, à la même époque que les tablettes de cire de Dijon, on fabrique des hanaps ou des manches de couteaux avec de l'érable appelé « madre ${ }^{16}$. L'érable de Pontarlier ${ }^{17}$ dans la comté voisine, peu éloignée de Cîteaux, était particulièrement prisé. Mahaut comtesse d'Artois et de la comté par alliance, achetait en Franche-Comté des objets ou des blocs de madre.

Cette possible relation entre les objets de bois dur et précieux et les tablettes de cire me semble intéressante à suivre et j'ai hâte de pouvoir faire analyser d'autres volumes de tablettes. Dans les textes des fabricants de tablettes de cire parisiens étudiés par Caroline Bourlet ${ }^{18}$, les tabletiers doivent utiliser du « bois blanc » et pour le support de l'écriture, de la cire et non du suif. Mais on trouve un peu partout dans la bibliographie un grand nombre de bois ${ }^{19}$ dont on faisait les tablettes: notamment le buis, bois lui aussi relativement dur.

L'identification du bois est importante pour connaître la place sociale des fabricants de tablettes: s'ils utilisent le madre, ce bois avec lequel on fabrique des hanaps et autres objets de luxe, ils peuvent figurer à juste titre à côté des orfèvres qui fabriquaient les carnets de tablettes

12. Élisabeth LALOU, «Inventaire des tablettes médiévales et présentation générale », dans Les tablettes à écrire de l'Antiquité..., p. 247

13. Une telle étude permettrait de répondre à une question posée par Martine Regert du C2RMF, spécialiste des cires : le bois était-il préparé pour recevoir la cire, était-il strié ?

14. Ce qui n'a pas été fait dans R. Bull, E. Moser, H. KuHN, « Wachs als Beschreib- und Siegelstoff... ». Les livres trouvés en Égypte au III ${ }^{e}$ siècle après J.-C. sont constitués de « Willow (silex) » c'est-à-dire de saule.

15. É. LALOU, Les comptes sur tablettes de cire de Jean Sarrazin..., p. 3 ; Natalis DE WAILLY, « Mémoire sur les tablettes de cire des Archives nationales ", Mémoire de l'Académie des Inscriptions et belles lettres 18, 1848, p. 536-563.

16. Il est difficile de trouver des exemples de hanap de madre. La vaisselle de bois est plus fréquemment mentionnée.

17. Françoise BARON, «Le mécénat de Mahaut d'Artois à Paris et en Artois », dans L'enfant oublié. Le gisant de Jean de Bourgogne et le mécénat de Mahaut d'Artois en franche Comté au XIVe siècle, Besançon, p. 44 (catalogue d'exposition du musée des Beaux-Arts et d'Archéologie de Besançon, décembre 1997-février 1998).

18. Caroline Bourlet, «Les tabletiers parisiens à la fin du Moyen Âge », dans Les tablettes à écrire de l'Antiquité..., p. 323-344.

19. Cèdre, brésil, cyprès, ébène, buis et encore ivoire et corne; cf. C. BOURLET, « Les tabletiers parisiens à la fin du Moyen Âge », dans Les Tablettes à écrire de l'Antiquité..., p. 326. 
d'ivoire dont nous avons conservé quelques feuillets. Si la grande majorité des tablettes de cire étaient fabriquées avec du bois plus commun, le métier entier des tabletiers perd un cran dans la hiérarchie des parisiens. Les deux possibilités pouvaient d'ailleurs co-exister.

Voici donc quelques pistes de recherche sur les matériaux utilisés pour les tablettes de cire. Identifier finement le bois et la cire utilisés devrait permettre de préciser le groupe social des fabricants comme des utilisateurs : on sait déjà que les tablettes de cire étaient d'une utilisation courante et que des milliers furent utilisées. Quoique très répandus, ces registres n'étaient peutêtre pas si humbles que cela. Ils étaient réservés à qui savait écrire.

L'identification des matériaux permettra aussi de préciser la relation avec le papier et son adoption - et le remplacement des tablettes de cire par des registres de papier, que je situe au milieu du XIV siècle pour la France. Je pense ici au prix comparé des tablettes de cire et du papier. Ce qui, étant donné la difficulté pour le Moyen Âge occidental de connaître les prix avec précision, est un projet très ambitieux. 